Journal of Patient-Centered

Volume 3

Issue 4 - Cardiovascular Aging

Article 10

$11-11-2016$

\title{
Predictors of Mortality in Patients With Transient Severe Left Ventricular Systolic Dysfunction
}

\author{
Kanwar Y. Singh \\ Firas Zahwe \\ Bilal Omery \\ Crystal Platz \\ Wassim Ballany \\ Robyn Shearer \\ Tadele Mengesha \\ M. Eyman Mortada \\ Jasbir Sra \\ Indrajit Choudhuri
}

Follow this and additional works at: https://aah.org/jpcrr

Part of the Cardiology Commons, and the Cardiovascular Diseases Commons

\section{Recommended Citation}

Singh KY, Zahwe F, Omery B, Platz C, Ballany W, Shearer R, Mengesha T, Mortada ME, Sra J, Choudhuri I. Predictors of mortality in patients with transient severe left ventricular systolic dysfunction. J Patient Cent Res Rev. 2016;3:235-6.

Published quarterly by Midwest-based health system Advocate Aurora Health and indexed in PubMed Central, the Journal of Patient-Centered Research and Reviews (JPCRR) is an open access, peer-reviewed medical journal focused on disseminating scholarly works devoted to improving patient-centered care practices, health outcomes, and the patient experience. 


\section{Proceedings of 2016 Aurora Scientific Day}

The following abstracts were presented at the 42nd annual Aurora Scientific Day research symposium, held May 25, 2016, in Milwaukee, Wisconsin. Aurora Scientific Day provides a forum for original research conducted by faculty, fellows, residents, students and other allied health professionals affiliated with Aurora Health Care, a not-for-profit health system comprised of integrated hospitals and clinics across eastern Wisconsin and northeastern Illinois.

\section{RIESELBACH DISTINGUISHED SESSION I Benefit of Report Card Feedback After Point-of-Care Assessment of Communication Quality Indicators}

Michael H. Farrell, Clair R. Sprenger, Shelbie L. Sullivan, Bree A. Trisler, Jessica J.F. Kram, Erin K. Ruppel

\section{Department of Family Medicine, Aurora UW Medical Group; Center for Urban Population Health}

Background: Communication in health care is crucial for patient experience and biomedical outcomes, but problems with communication are often seen in health care. Training can improve communication, but skills must be reinforced after graduation to remain improved. Since educational methods are too resource intensive for sustained use throughout the Aurora Health Care system, it is necessary to develop affordable, quantitative methods. The first author has developed necessary techniques, including behavior-specific measures called communication quality indicators.

Purpose: To demonstrate secure audio recording in an outpatient visit and to use communication quality indicators with a heterogenous set of patient-clinician conversations.

Methods: Thirty primary care physicians were audio-recorded with one or more patients via a secure Internet application running on exam-room computers. Transcripts were abstracted quantitatively using explicit-criteria definitions for two groups of communication quality indicators: assessments of understanding (AU) and jargon explanations (JE). There are four separate behaviors within the AU group: open-ended, close-ended, the highly effective "request for teachback," and the least effective "OK?" question. Quality indicator data were returned using a previously described report card. After feedback, one or more follow-up recordings were done for comparison.

Results: Baseline transcripts included a mean of 15.5 unique jargon words, but words were often used more than once so the mean total jargon count was 25.1. JEs were rare at baseline, with a median of 1 per transcript. The JE ratio (fraction of jargon words that follow a JE for that word) averaged 0.26 out of a best-possible 1.0. AUs were found in $61.1 \%$ of transcripts, but most were "OK?" (median 2.13/transcript) or close-ended questions (median 0.52/transcript). After the report card, the median number of JEs improved to 4 per transcript $(\mathrm{P}<0.01$ by Wilcoxon), and the JE ratio improved to 0.36 ( $\mathrm{P}<0.01$ by matched t-test). AUs improved to $81.3 \%$ of transcripts $(\mathrm{P}<0.04$ by chi-squared). Most of the increase was found in close-ended
AUs (median 0.97/transcript by, $\mathrm{P}<0.04$ by Wilcoxon).

Conclusion: This project demonstrated that it is feasible to record at the point of care, abstract transcripts at a central office and improve communication quality via a report card. The small sample size was acceptable for a demonstration project, but a larger, multifaceted program could improve patient experience and biomedical outcomes across Aurora.

RIESELBACH DISTINGUISHED SESSION II Predictors of Mortality in Patients With Transient Severe Left Ventricular Systolic Dysfunction

Kanwar Y. Singh, Firas Zahwe, Bilal Omery, Crystal Platz, Wassim Ballany, Robyn Shearer, Tadele Mengesha, M. Eyman Mortada, Jasbir Sra, Indrajit Choudhuri

\section{Aurora Cardiovascular Services, Aurora Health Care; Aurora Research Institute}

Background: About $20 \%$ of patients who develop left ventricular (LV) systolic dysfunction will have improvement in ejection fraction (LVEF) over time. This patient cohort is generally excluded from large sudden death trials and, hence, understudied.

Purpose: To evaluate the predictors of mortality in patients with severe LV systolic dysfunction who have improvement in LVEF during follow-up.

Methods: Patients who had transient LV systolic dysfunction from 2010 to 2014 within the Aurora Health Care system and who had LVEF improve to $\geq 40 \%$, irrespective of implantable cardioverter-defibrillator (ICD) implant, were studied. Predictors of mortality were identified using Cox proportional hazards model. Patients were then divided into groups based on LVEF $>50 \%$ or $<50 \%$ to assess for benefit of ICD using Kaplan-Meier estimates.

Results: A total of 1,364 patients met inclusion criteria; $58.4 \%$ were male, and mean BMI was $29 \pm 7$. Mean age postLVEF improvement was $66 \pm 14$ years, and with each added year the hazard rate increased by $5 \%$ (hazard ratio [HR]: $1.05, \mathrm{P}<0.0001)$. Several clinical characteristics emerged as predictors of mortality, including smoking (HR: 1.8, $\mathrm{P}=0.0002$ ), chronic renal disease (HR: 2.3, $\mathrm{P}<0.0001$ ), atrial fibrillation (HR: 1.4, $\mathrm{P}=0.013$ ) and no-ICD (HR: 2.1, $\mathrm{P}=0.012$ ). With each percentage increase in LVEF, hazard rate decreased by $2 \%$ (HR: $0.97, \mathrm{P}=0.007)$. However, presence of ICD did not significantly improve mortality in the group with LVEF $>50 \%$ 
$(\mathrm{P}=0.2)$, whereas it continued to show benefit in the group with LVEF of $40 \%-49 \%(\mathrm{P}=0.001)$.

Conclusion: Clinical predictors of mortality in patients with transient LV systolic dysfunction may help further risk-stratify this cohort of patients. It appears that patients with LVEF of $40 \%-49 \%$ continue to derive benefit from ICD therapy.

\section{FIRST PLACE ORAL PRESENTATION}

See page 245 for citation.

\section{SECOND PLACE ORAL PRESENTATION Path to Resistance: Risk Factors Associated With Carbapenem-Resistant Pseudomonas aeruginosa}

\author{
Kushal Patel, Jessica J.F. Kram, Dennis J. Baumgardner
}

Department of Internal Medicine, Aurora Sinai Medical Center; Department of Family Medicine, Aurora UW Medical Group; Center for Urban Population Health

Background: An estimated 51,000 health care-associated Pseudomonas aeruginosa infections occur in the United States annually. More than $13 \%$ are secondary to non-carbapenem multidrug-resistant strains, which result in 400 yearly deaths. Traditional risk factors for resistance include ICU stay, mechanical ventilation, previous hospitalization and major comorbidities. As microbes evolve, risk factors also may evolve.

Purpose: To determine if traditional and/or new risk factors for $P$. aeruginosa resistance are valid and predictive of infection with carbapenem-resistant $P$. aeruginosa.

Methods: We retrospectively studied inpatients and outpatients $\geq 18$ years old who presented to an Aurora Health Care facility with a positive $P$. aeruginosa culture during 2014. Cultures were obtained from the ACL Laboratories database, and patient medical records were reviewed in Epic. Chi-squared test with Yates correction and two-sample t-tests were performed on categorical and continuous variables, respectively. Binary regression was used for multivariable modeling. Significance was associated with $\mathrm{P}<0.05$.

Results: Study population $(\mathrm{N}=1,763)$ characteristics were: mean age 68.0 , body mass index $30.4 \mathrm{~kg} / \mathrm{m}^{2}, 51.2 \%$ female sex, and $89.3 \%$ white race. Resistance to imipenem or meropenem $(14.0 \%)$ on univariable analysis was associated with younger age ( 66.0 vs 68.3 years, $\mathrm{P}=0.027)$, hospitalized patients $(19.7 \%$ vs $8.6 \%, \mathrm{P}<0.0001)$, male sex $(16.0 \%$ vs $12.0 \%, \mathrm{P}=0.017)$, nonwhite race $(23.5 \%$ vs $12.3 \%, \mathrm{P}<0.0001)$, respiratory culture $(30.9 \%$ vs $12.1 \%, \mathrm{P}<0.0001)$, history of pulmonary disease $(19.4 \%$ vs $12.9 \%, \mathrm{P}=0.005)$, history of congestive heart failure ( $18.6 \%$ vs $13.0 \%, \mathrm{P}=0.016)$, history of multidrug resistance $(33.3 \%$ vs $13.6 \%, \mathrm{P}=0.003)$ and recent surgery $(17.8 \%$ vs $12.2 \%, \mathrm{P}=0.002)$, as well as transfer from institution, Foley catheter, vasopressor treatment, central/PIC lines, mechanical ventilation, ICU admission, and bedridden status (all $\mathrm{P}<0.0001$ ). In multivariable modeling, nonwhite race, respiratory culture, recent transfer, vasopressor use and central/PIC lines were significant. Only $0.57 \%$ of strains were resistant to the six traditional non-carbapenem drugs and both carbapenems.

Conclusion: Demographic and traditional risk factors, as well as respiratory cultures, were predictive of carbapenem resistance. Such information may guide initial antibiotic treatment of $P$. aeruginosa. Fortunately, less than $1 \%$ of strains were resistant to all drugs tested. Further studies looking at change in outcome while incorporating these risk factors in determination of empiric coverage for patients should be performed.

\section{THIRD PLACE ORAL PRESENTATION}

See page 245 for citation.

\section{FIRST PLACE POSTER}

See page 245 for citation.

\section{SECOND PLACE POSTER (tie)}

The Association Between Doppler Measures of Cardiac Function and Outcomes in Patients With Left Ventricular Ejection Fraction $\leq \mathbf{4 0} \%$ Undergoing Noncardiovascular Surgeries

Yang Shi, Rachel Pedersen, Matthew Rappelt, Robyn Shearer, Nasir Z. Sulemanjee, Dianne L. Zwicke, T. Edward Hastings, Omar M. Cheema, Vinay Thohan

Sheikh Khalifa bin Hamad Al Thani Center for Integrative Research on Cardiovascular Aging, Aurora Research Institute; Transplant Administration, Aurora Health Care; Aurora Cardiovascular Services, Aurora Health Care

Background: Preoperative risk assessments of individuals who undergo major noncardiac surgery have focused on ischemic heart disease. Information on how to assess the noncardiac surgical risks for patients with depressed cardiac function, as seen in heart failure, is sparse. Echocardiography is routinely performed in patients with depressed cardiac function and is an accepted standard cardiac assessment. Transthoracic echocardiography (TTE) provides strong independent prognostic implications in a wide range of cardiovascular conditions.

Purpose: To identify the echocardiographic parameters associated with outcomes among patients undergoing major noncardiac surgery.

Methods: A retrospective single-institution investigation identified 1,770 patients who underwent one or more major noncardiac procedures from Jan. 1, 2011, to June 30, 2014, and had at least one TTE performed within 90 days before surgery. Patients were stratified by presurgery left ventricular ejection fraction (LVEF) into LVEF $\leq 40 \%$ and LVEF $>40 \%$ groups. The cohort was followed through June 12, 2015, with the outcome focused on all-cause mortality. Continuous and categorical variables were compared by Student's t-test and chi-squared test, respectively. Kaplan-Meier method was used to calculate mortality estimates postsurgery. Cox proportional hazards model was used for univariate and multivariable models. 